\title{
Study of pulse shape discrimination for a neutron phoswich detector
}

\author{
Jessica Hartman $^{1}$ and Alexander Barzilov ${ }^{1}$ \\ ${ }^{1}$ Department of Mechanical Engineering, University of Nevada, Las Vegas, 4505 S. Maryland Parkway, Las Vegas, NV, 89154, USA
}

\begin{abstract}
A portable phoswich detector capable of differentiating between fast neutrons and thermal neutrons, and photons was developed. The detector design is based on the use of two solid-state scintillators with dissimilar scintillation time properties coupled with a single optical sensor: a ${ }^{6} \mathrm{Li}$ loaded glass and EJ299-33A plastic. The on-the-fly digital pulse shape discrimination and the wavelet treatment of measured waveforms were employed in the data analysis. The instrument enabled neutron spectrum evaluation.
\end{abstract}

\section{Introduction}

Nuclear technologies are utilized in power industry, medical isotope production, food irradiation, scanning of cargo, and other applications. The worldwide expansion of nuclear technologies calls for portable instruments that enable detection and quantification of radiation sources and materials. The development of instruments capable of fast neutron, thermal neutron, and photon detection is an ongoing effort [1]. The ability for simultaneous analysis of these three signatures provides an additional level of certainty in the radiation assay. Besides, neutron energy is a unique signature for nuclear material or radiation source identification. Previous development efforts were focused primarily on the application of the multi-scintillator design to gamma-ray, alpha- and beta-particle measurements $[2,3]$.

To meet the goal of the neutron source detection and its identification, this research project was focused on the development of a portable phosphor sandwich (phoswich) detector capable of differentiating between fast neutrons, thermal neutrons, and photons. A neutron phoswich design is based on the use of two scintillators with dissimilar times of scintillation coupled with a single optical sensor - a photomultiplier tube (PMT).

\section{Phoswich detector}

The instrument employs two solid-state scintillators with the dissimilar properties of the scintillation light decay. The first scintillator is a glass disc $(5-\mathrm{cm}$ in diameter and 1 -mm thick) loaded with $1.58 \times 10^{22}$ of ${ }^{6} \mathrm{Li}$ atoms $/ \mathrm{cm}^{3}$ enabling high sensitivity to thermal and epithermal neutrons, and a low sensitivity to high energy photons and fast neutrons [4]. The thermal-neutron-induced scintillation decay time for this cell is about $60 \mathrm{~ns}$. The second scintillator is a cylindrical cell $(5-\mathrm{cm}$ in diameter and 5-cm long) of the EJ-299-33A plastic.
This plastic offers pulse shape discrimination (PSD) properties which enable the differentiation between neutrons and gamma rays [5]. It has a scintillation time constant of about $4 \mathrm{~ns}$ for photons, and about $30 \mathrm{~ns}$ for the neutrons through the proton recoil. Two scintillator cells were coupled to a single PMT. The computer controlled high-voltage (HV) base was used to supply power to the PMT. The photomultiplier's anodet signals were processed with a DAQ chip - a miniature digitizer eMorpho (Bridgeport Instruments). The digitizer was connected to a small Linux computer (BeagleBone) equipped with a LCD touchscreen. A WiFi module was used for the wireless communication. A rechargeable Liion battery was utilized for portability of the system. The layout of the instrument is shown in Fig. 1. The dimensions of the detector are $9 \mathrm{~cm} \times 25 \mathrm{~cm} \times 17 \mathrm{~cm}$. The portable detector module is shown in Fig. 2.

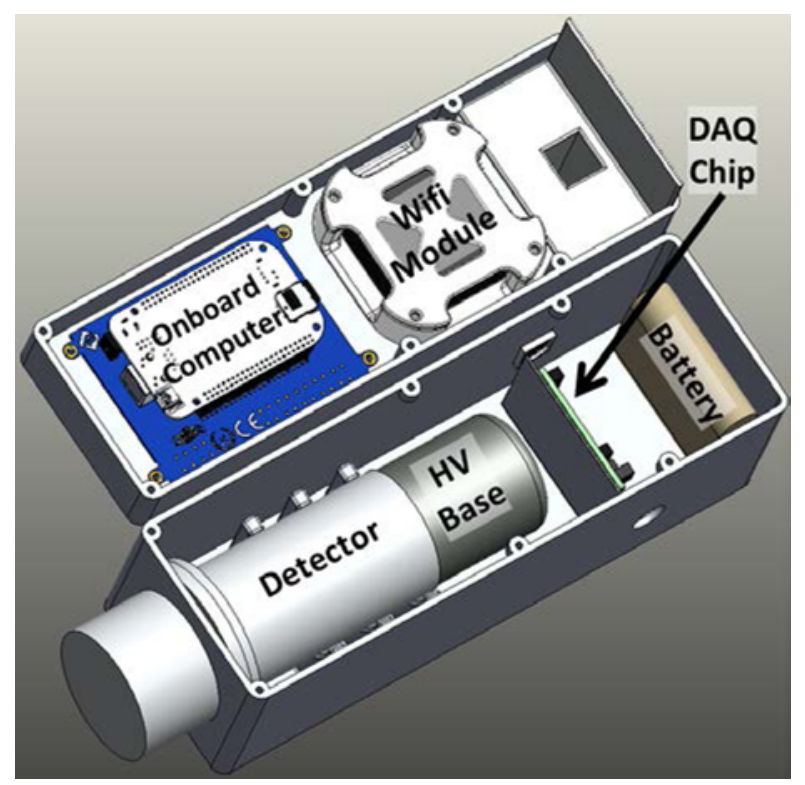

Fig. 1. Internal layout of the instrument. 


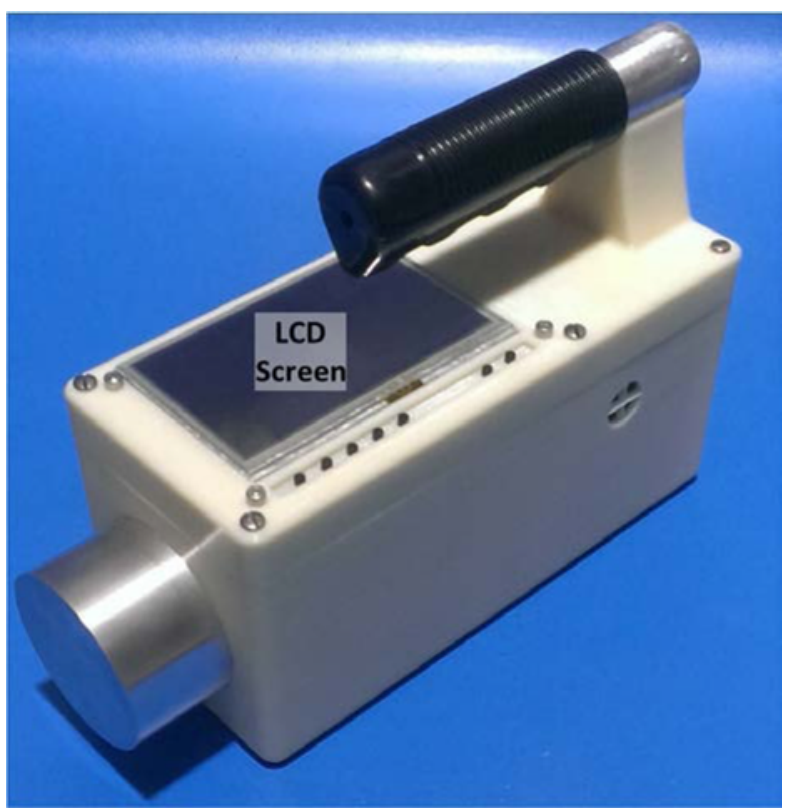

Fig. 2. Portable detector module.

The scheme of the phoswich operation is shown in Fig. 3. Incident radiation emitted from a source enters the phoswich assembly, reacting with the scintillator cells to produce scintillation photons. The PMT anode signals generated due to these reactions are sent to the DAQ chip. This digitizer processes the data using the list mode and assigns the energy, time, and particle identification (PID) values to each of the incoming signals.

The PID value is a ratio of the integral under the tail of the signal to the integral of the front part of the pulse. The PID enables segregating waveforms generated by photons, thermal neutron, and fast neutrons. The IT and PIT integration time values (see Fig. 4) were set up to achieve the optimal figure of merit (FOM) of the PSD.

Processed signals are then sent to the onboard computer, where the PID value is used to carry out the on-the-fly PSD analysis thus separating the signals caused by different incident radiations.

Once the digital waveforms were segregated, a wavelet treatment (Eq. 1) was applied to the waveforms $R(E)$ using the scaling function $\varphi$ with unique scale $(a)$ and shift (b) parameters, generating a wavelet $\Psi_{n}$ which accurately plots the shape of the response while smoothing the data:

$$
\Psi_{n}=\int_{-\infty}^{\infty} R(E)\left(\frac{1}{\sqrt{a}}\right) \varphi\left(\frac{E-a}{b}\right) d E
$$

The inherent ability of the wavelets to maintain key signal features while reducing noise sensitivity during fitting procedures makes it ideal for use in the spectral unfolding.

A least squares regression technique was utilized for decomposition of a polyenergetic neutron detector response as the sum of monoenergetic neutron responses. The discrete form of the Fredholm integral was utilized (Eq. 2).

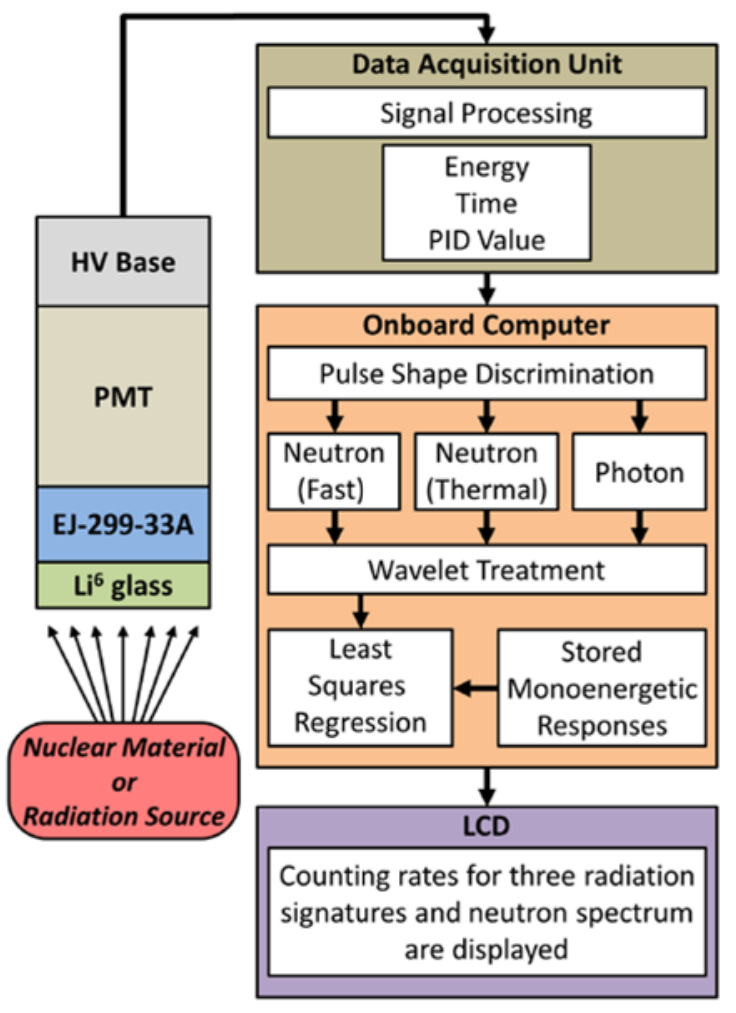

Fig. 3. Scheme of operation of a phoswich detector.

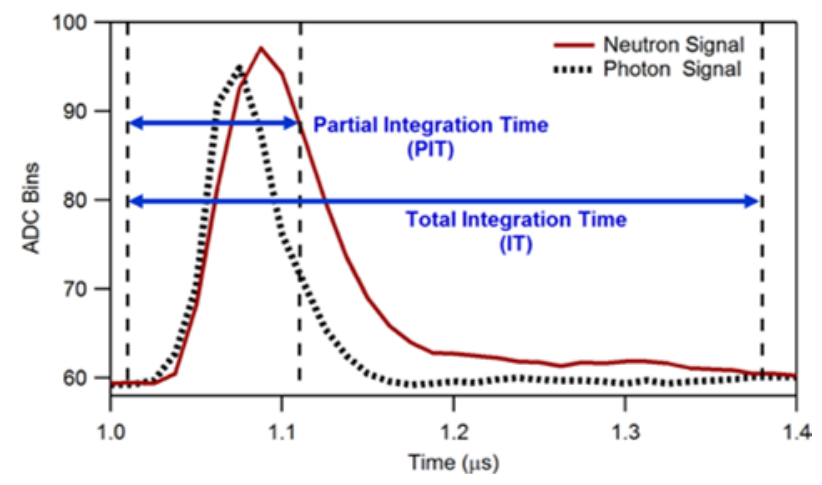

Fig. 4. Digitized PMT signal waveforms for the EJ-299-33A scintillator cell.

$$
N_{i}+e_{i}=\sum_{j} R_{i j} S_{j}
$$

where $N_{i}$ is the data for a channel $i, e_{i}$ is a discrepancy between the reconstructed and measured values, $R_{i j}$ is the monoenergetic response for neutron energy $j$, and $S_{j}$ is a weight.

The responses $R_{i j}$ were measured using acceleratorbased monoenergetic neutron sources and stored as a data library. Gaseous tritium and deuterium targets with incident proton and deuteron beams were used. $T(p, n)^{3} \mathrm{He}, D(d, n)^{3} \mathrm{He}$, and $T(d, n)^{4} \mathrm{He}$ reactions were employed to generate mono-energetic neutrons. The angular dependence of the neutron yield for these reactions [6] allowed measurements of response functions for neutron energies ranging from $0.1 \mathrm{MeV}$ to $8 \mathrm{MeV}$ and from $12 \mathrm{MeV}$ to $20 \mathrm{MeV}$. 
The code supplied with the math libraries was used to solve Eq. 2 for the weights $S_{j}$ by minimization of the error $e_{i}$. The weights represent the unfolded neutron spectrum.

\section{Results}

PSD capabilities of the phoswich system were experimentally evaluated. Fig. 5 shows segregated fast neutron and gamma-ray signals measured using the plastic cell and a 2-Ci $\mathrm{PuBe}$ source. The slower scintillation decay time for the ${ }^{6} \mathrm{Li}$-loaded cell also allowed measuring the thermal-neutron count rate.

The PSD FOM was determined using full width half maximum values and the separation between neutron and photon distributions shown in the plot of count rates versus the particle ID values (Fig. 6). The FOM was measured as 1.3 for the plastic scintillator cell.

The unfolded neutron spectra for deuteriumdeuterium (DD) and deuterium-tritium (DT) neutron sources are shown in Fig. 7. The spectral unfolding technique did show sensitivity to the stored detector response functions.

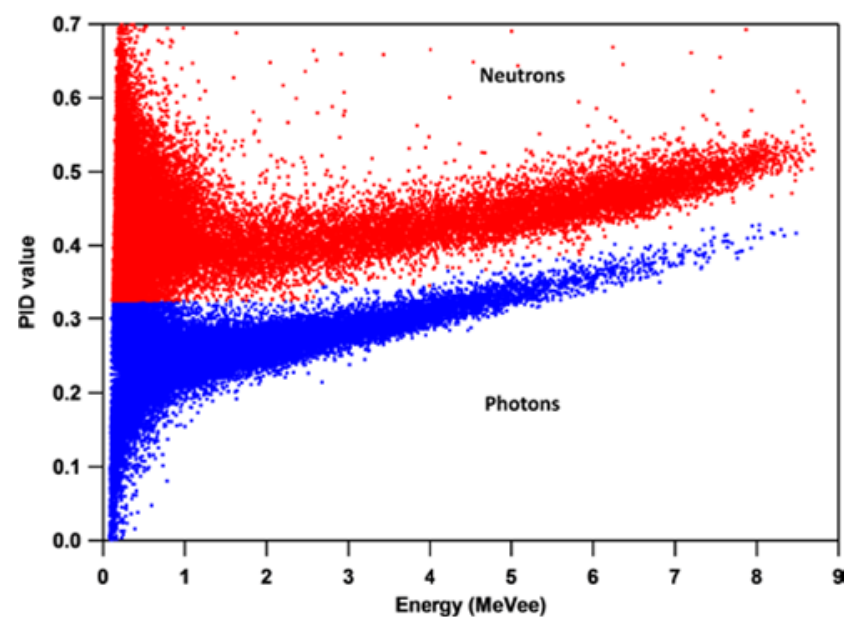

Fig. 5. PSD plot for the EJ-299-33A cell.

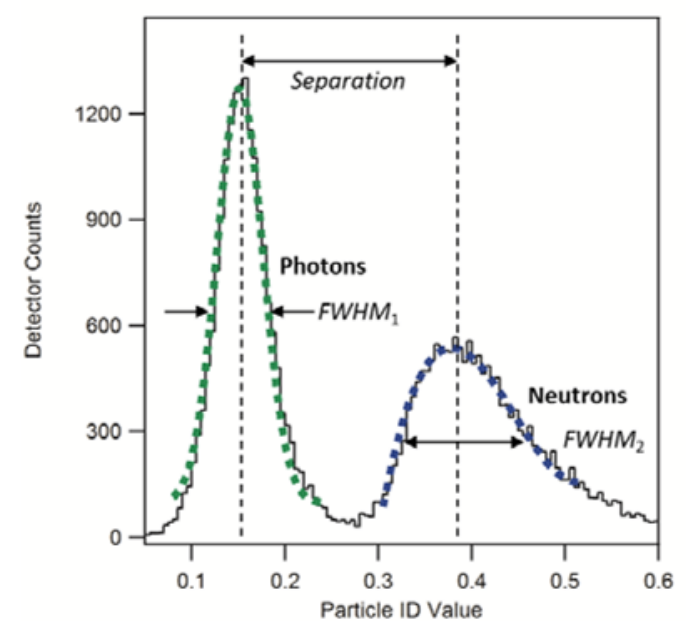

Fig. 6. Neutron/photon FOM.

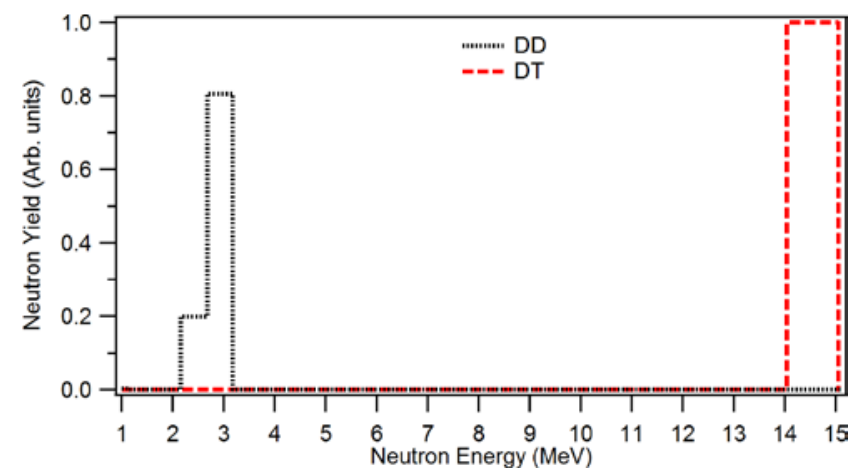

Fig. 7. Unfolded spectra for DD and DT neutron sources.

\section{Conclusions}

A phoswich neutron detector of the small form factor was designed. The detector enabled onboard data analysis with a wireless communication of the measured data. The phoswich was capable of simultaneously measuring gamma-ray, thermal neutron, and fast neutron count rates, and the fast-neutron spectrum evaluation. The results were reported on the LCD screen, displaying count rates for radiation signatures and the unfolded neutron spectrum.

\section{References}

1. N. Zaitseva, A. Glenn, H.P. Martinex, L. Carman, I. Pawelczak, M. Faust, S. Payne, "Pulse shape discrimination with lithium-containing organic scintillators," Nucl. Instrum. Meth., 729, 747 (2013).

2. S. Usuda, S. Sakurai, K. Yasuda, "Phoswich detectors for simultaneious counting of $\alpha-\beta(\gamma)$-rays and neutrons," Nucl. Instrum. Meth., 388, 193 (1997).

3. S. Yamamoto, H. Ishibashi, "Development of a three-layer phoswich alpha-beta-gamma imaging detector," Nucl. Instrum. Meth., 785, 129 (2015).

4. L. Greenwood, N. Chellew, G. Zarwell, "Comparative analysis of pulse shape discrimination methods in a ${ }^{6} \mathrm{Li}$ loaded plastic scintillator," Nucl. Instrum. Meth., 788, 146 (2015).

5. Eljen Tech: http://www.eljentechnology.com.

6. H. Liskien, A. Paulsen, "Neutron production cross sections and energies for the reactions $\mathrm{T}(\mathrm{p}, \mathrm{n})^{3} \mathrm{He}$, $\mathrm{D}(\mathrm{d}, \mathrm{n})^{3} \mathrm{He}$, and $\mathrm{T}(\mathrm{d}, \mathrm{n})^{4} \mathrm{He}$, " Atomic Data and Nuclear Data Tables (1973). 